OPEN

SUBJECT AREAS:

STATISTICS

ECOLOGY

THEORY

STATISTICAL PHYSICS, THERMODYNAMICS AND NONLINEAR DYNAMICS

Received

9 March 2012

Accepted

27 June 2012

Published

11 July 2012

Correspondence and requests for materials should be addressed to

B.M.W. (bmweon@ hotmail.com) or J.H.J. (jhje@postech.ac.kr)

\section{Trends in scale and shape of survival} curves

\author{
Byung Mook Weon \& Jung Ho Je
}

X-ray Imaging Center, Department of Materials Science and Engineering, Pohang University of Science and Technology, San 31 , Hyoja-dong, Pohang, 790-784, Korea.

The ageing of the population is an issue in wealthy countries worldwide because of increasing costs for health care and welfare. Survival curves taken from demographic life tables may help shed light on the hypotheses that humans are living longer and that human populations are growing older. We describe a methodology that enables us to obtain separate measurements of scale and shape variances in survival curves. Specifically, 'living longer' is associated with the scale variance of survival curves, whereas 'growing older' is associated with the shape variance. We show how the scale and shape of survival curves have changed over time during recent decades, based on period and cohort female life tables for selected wealthy countries. Our methodology will be useful for performing better tracking of ageing statistics and it is possible that this methodology can help identify the causes of current trends in human ageing.

U nderstanding the reasons for the evolution of senescence is of great importance for investigating the ageing mechanisms of humans and other organisms ${ }^{1,2}$. Demography is an essential tool in ageing research, in conjunction with evolution, ecology, and medicine, because more information is available on humans than on any other species ${ }^{3-5}$. Demographic data contain thousands of records, repeated measurements on individuals, considerable environmental information and even complete genealogies ${ }^{3}$. Such data can be used to test evolutionary hypotheses, such as the role of parental care in the evolution of post-reproductive life, which cannot be tested on other species ${ }^{4}$. Determining the appropriate mathematics for representing survival curves is a key topic in demography and population biology relative to the quantification and prediction of ageing trends. The survival function, $s(x)$, is typically represented as a monotonically decreasing function that declines from 1 to 0 as age increases. The mortality function (hazard function or force of mortality) is mathematically linked to the survival function and is defined as a negative derivative of the logarithmic survival rate, namely, $\mu(x)=$ $-\mathrm{d} \ln (s(x)) / \mathrm{d} x$. In 1825, Gompertz found that human mortality rates increase exponentially with age ${ }^{6}$. This exponential kinetics is indicative of a progressive degenerative process and is observed in many organisms, including mammals, flies, worms, plants, and yeast cells ${ }^{7}$. This feature has been explained in terms of a decline in the force of natural selection acting on age-specific mortality ${ }^{8}$. The Gompertz law is expressed as $\mu(x)=a$ $\exp (b x)$ or $\ln (\mu(x))=\ln (a)+b x$, where the age-specific mortality rate, $\mu(x)$, is determined by two parameters: $\ln (a)$ and $b$. In 1960, Strehler and Mildvan found a correlation between the two Gompertz parameters ${ }^{9}$. According to the Strehler-Mildvan law, $\ln (a)$ and $b$ are negatively correlated in the relation $\ln (a)=\ln (\mathrm{K})-\mathrm{B}^{-1} b$, where $\mathrm{K}$ is the frequency of environmental stresses and $\mathrm{B}$ is the slope of the age-related decline in individual physiological capacities $^{10,11}$.

We recently developed a mathematical method to explain the response of the scale and shape of survival curves to the shift of the survival curve towards an ideal rectangular curve for human and non-human populations $s^{12-14}$. The main feature of our method is the adoption of a modified stretched exponential survival function, typically described as $s(u)=\exp \left(-u^{\beta(u)}\right)$, where $u$ is the rescaled age taken by dividing the age $(x)$ by the characteristic life $(\alpha)$ and $\beta(u)$ is the age-dependent stretched exponent given by $\ln [-\ln (s(u))] / \ln (u)$. A critical difference between our function and the classical stretched exponential (also known as the Kohlrausch-Williams-Watts [KWW ${ }^{15}$ or Weibull ${ }^{16}$ ) function is the age dependence of the stretched exponent ${ }^{14}$. In a survival curve, $\alpha$ indicates the scale effect and $\beta$ indicates the shape effect (ref. 14). The scale effect on the characteristic life can be ruled out by rescaling the age as $u=x / \alpha$, enabling us to quantify the shape effect of a survival curve. The shape effect is relevant to the change in the curvature of a survival curve. When a healthy population achieves a rectangular-like survival curve, $\beta(u)$ tends to shift towards an ideal curve of $\beta(u)=7|\ln (u)|^{-1}$, corresponding to an ideal rectangular survival curve of $s(u<1) \approx 0.999089$ and $s(u>1) \approx 0$ (ref. 14). From the measurement of $\alpha$ at $s(\alpha)=\exp (-1) \approx$ 0.367879 and the determination of $\beta(u)$, an exact formula for the mortality rate can be obtained as $\mu(u)=(u \beta(u) / \alpha)$ $[\beta(u) / u+\ln (u) \mathrm{d} \beta(u) / \mathrm{d} u]($ ref. 14$)$. 
a

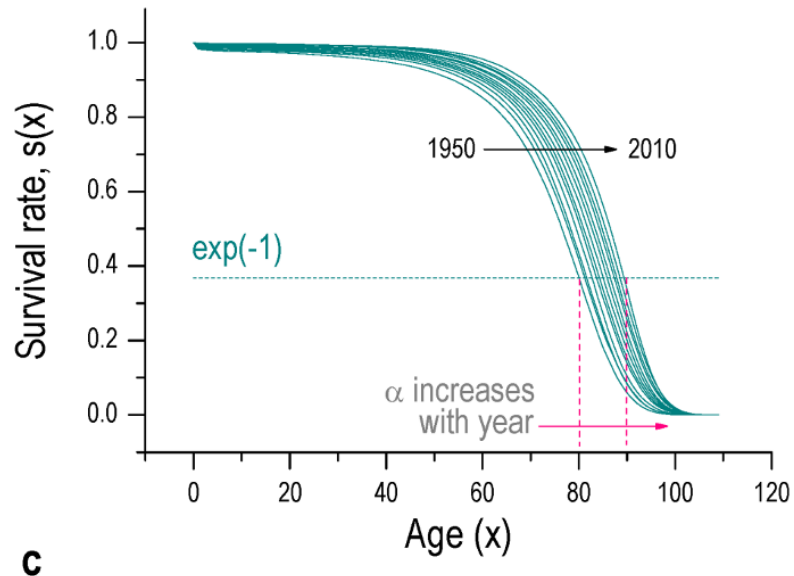

c

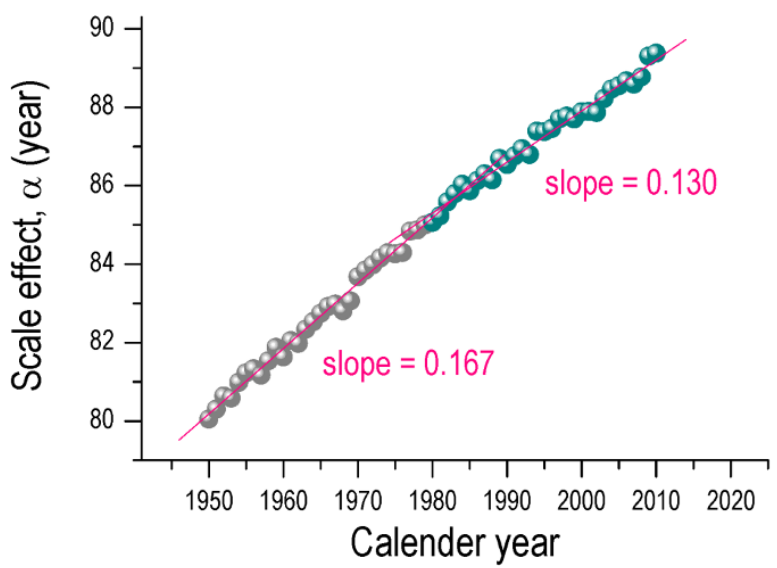

b
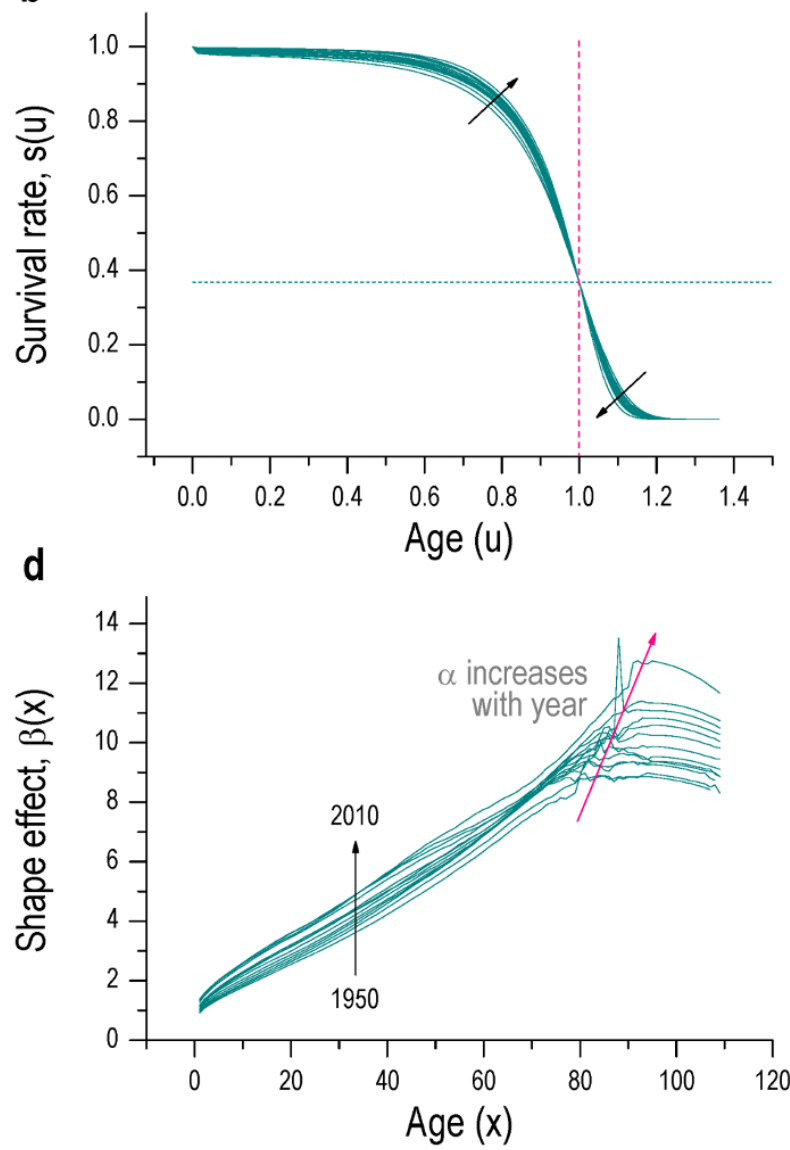

Figure $1 \mid$ Methodology for scale and shape analyses of survival curves. (a) The historic trends in survival curves, $s(x)$, for Swedish females during the past six decades $(1950-2010$; interval $=5$ years $)$ show that $s(x)$ gradually shifts rightwards and towards a rectangular shape. The characteristic life $(\alpha)$ for each survival curve was accurately measured by detecting the interception point between $s(x)$ (solid line) and $s(\alpha)=\exp (-1)($ dashed line). The scale effect, revealed by the $\alpha$ value, can be removed by rescaling the age with $u=x / \alpha$, enabling us to quantify how survival curves become rectangular as populations become older. (b) The rescaled survival curves show a tendency to bend towards the $u=1$ axis (the bending is depicted by arrows). (c) The historical trends in the characteristic life, $\alpha$, (scale effect) show that the slope decreases slightly from 0.167 (1.67 years per decade for 1950-1979) to 0.130 (1.30 years per decade for $1980-2010$ ). (d) The historical trends in the stretched exponent, $\beta(x)$, (shape effect) show that $\beta(x)$ gradually shifts rightwards and upwards from 1950 to 2010 as $\alpha$ increases with the calendar year.

In this study, we investigate human survival and death (mortality) rate trends with a stretched exponential survival function. In these investigations, scale and shape effects in survival curves are evaluated by separately measuring the characteristic life and the stretched exponent. Specifically, 'living longer' is relevant to the scale variance in survival curves, whereas 'growing older' is relevant to the shape variance. Based on period and cohort female demographic life tables for selected wealthy countries, we show how the scale and shape of survival curves has changed over time during recent decades, particularly after World War II. The current trends in the survival curves show interesting results: the scale effect (characteristic life) linearly increases over time, indicating 'living longer', and the shape effect (stretched exponent) gradually shifts towards rectangularisation, indicating 'growing older'. Most ageing studies have focused on death (mortality) rate patterns ${ }^{17-22}$, but important implications of survival curves may be overlooked without scale and shape analyses of these curves. Our methodology could be useful for performing better tracking of ageing statistics, and it is also possible that this methodology can help identify the causes of current trends in human ageing.

\section{Results}

Scale and shape analyses of survival curves. Human survival curves have shown dramatic trends during recent decades. Our methodology of analysing these curves, as based on Swedish female period life tables from 1950 to 2010, is demonstrated in Fig. 1. The survival curves were obtained from reliable statistical data in the Human Mortality Database (http://www.mortality.org), taking $s(x)=10^{-5} l_{x}$ (in life tables, $l_{x}$ means the number of survivors at age $(x)$ normalised by 100,000 people). Current trends in survival curves are evaluated by analysing the characteristic life $(\alpha)$ corresponding to a singular age at $s(\alpha)=\exp (-1) \approx 0.367879$ and by analysing the stretched exponent $\beta(x)$. The historic trends in survival curves can be quantified in terms of the scale and shape effects, described by $\alpha$ and $\beta(x)$, respectively. For instance, the scale effect is revealed by the gradual increase in the characteristic life, as described by the arrow in Fig. 1a, and the shape effect can be visualised by the cancellation of the scale effect (that is, by rescaling the age with the characteristic life), as demonstrated in Fig. 1b. Here, the cancellation of the scale effect is useful to evaluate how survival curves become rectangular as populations grow older. In fact, the survival curves for Swedish female period data are bent towards the $u=1$ axis (the bending is depicted by arrows in Fig. 1b), implying that the ages of the older people in the population are concentrated in an increasingly narrow range. A shape analysis of this type would facilitate the qualitative identification of the rectangularisation of the survival curves. Note that the survival curves tend to shift rightwards by the scale effect, indicating 'living longer', and towards the asymptotic axis of $u(=x / \alpha)$ $=1$ by the shape effect, indicating 'growing older'. 

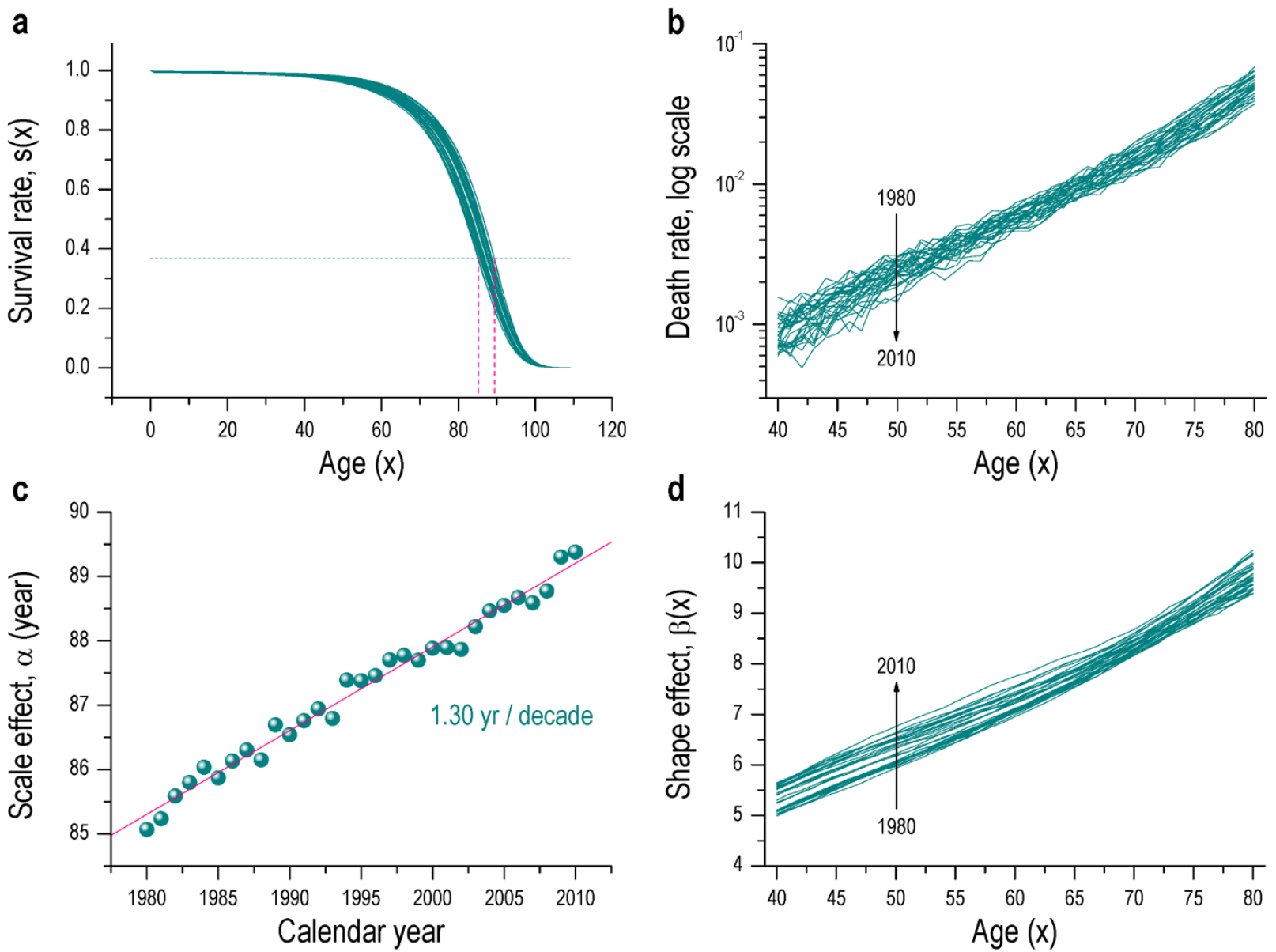

Figure $2 \mid$ Historical trends for Swedish female period data. The death (mortality) rate trends must be associated with the survival rate trends because they are mathematically linked. (a) The historical trends from 1980 to 2010 in the survival rates for Swedish female period data. (b) The historical trends in death rates, $\mu(x)$, at 40-80 years show exponential increases with age, suggesting that the Gompertz law would be valid for these datasets. (c) The scale effect $(\alpha)$ increases over time at a constant rate of 1.30 years per decade from 1980 to 2010 . (d) The shape effect, $\beta(x)$, at $40-80$ years increases linearly with age. This trend strongly resembles the linear growth in $\ln (\mu(x))$ with age (b) except for the shift direction: "upwards" in $\beta(x)$ and "downwards" in $\ln (\mu(x))$. The strong correlation between $\ln (\mu(x))$ and $\beta(x)$ implies that death rates are coupled with survival rates and are susceptible to the shape effect in survival curves.

To evaluate current trends in terms of the scale variance of survival curves, the characteristic life in each survival curve was accurately measured by detecting the interception point between $s(x)$ and $s(\alpha)$ $=\exp (-1)$, as depicted by the dashed lines in Fig. 1a (Supplementary Table S1; a standard deviation in graphical measurements $\approx 3 \times 10^{-5}$ ). The $\alpha$ value can be a good measure of the scale effect as an alternative to life expectancy at birth $(\varepsilon)$ (Supplementary Fig. S1). The trends of the characteristic life for the Swedish female period data show an upwards increase with the calendar year during the past six decades (Fig. 1c). We find a significant deceleration in the increase of the characteristic life: the slope decreases from 0.167 (1.67 years per decade) for 1950-1979 to 0.130 (1.30 years per decade) for 1980-2010. This deceleration is similarly found in the life expectancy at birth for Swedish women, from 0.207 for $1950-1979$ to 0.151 for $1980-2010$ (Supplementary Fig. S1).

To gain insights in terms of the shape variance of survival curves, we consider the trends for the stretched exponent. In our method, using $s(x)=\exp \left(-(x / \alpha)^{\beta(x)}\right)$, the stretched exponent can be measured from $\beta(x)=\ln [-\ln (s(x))] / \ln (x / \alpha)$ for normal age $(x)$. This exponent is important in the assessment of the rectangularity of a survival curve because it allows the evaluation of the shift of $\beta(u)$ towards the ideal curve of $\beta(u)=7|\ln (u)|^{-1}$. This ideal curve corresponds to the ideal survival curve of $s(u<1) \approx 1$ and $s(u>1) \approx 0$ (ref. 14). We illustrate the historic trends in $\beta(x)$ from 1950 to 2010 in Fig. 1d. This figure shows that $\beta(x)$ increases with age and shifts upwards with the calendar year. This shift implies that the survival curves become more rectangular, i.e., more people are growing older. Without rescaling the age by the characteristic life, the historical trends in $\beta(x)$ show that $\beta(x)$ shifts rightwards and upwards in parallel with the increase in the characteristic life. This result suggests that the scale effect significantly influences the shape effect in survival curves and confirms that humans are living longer and that human populations are growing older.

Correlation between survival and death rates. We compare trends in the scale and shape of survival curves with death rate trends. We focus on the most recent three decades (after 1980) to rule out the slope transition at 1980 in the characteristic life (see Fig. 1c). We illustrate the historic trends from 1980 to 2010 in survival rates (Fig. 2a) and in death rates at 40-80 years (Fig. 2b) for the Swedish female period data taken from the Human Mortality Database. Trends in death (mortality) rates must be associated with trends in survival rates because death and survival functions are mathematically linked.

The death rates, $\mu(x)$, at $40-80$ years (Fig. $2 \mathrm{~b}$ ) exponentially increase with age, suggesting that the Gompertz law would be valid for Swedish female period data from 1980 to 2010. From simple linear regressions between $\ln (\mu(x))$ and $x$ according to the Gompertz law as $\ln (\mu(x))=$ $\ln (a)+b x$, we measured the intercept $(\ln (a))$ and the slope $(b)$ for 
a

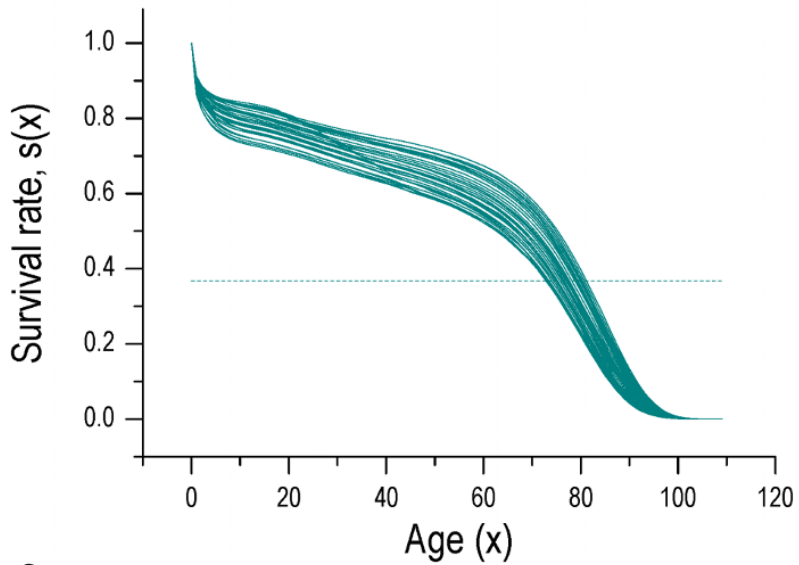

C

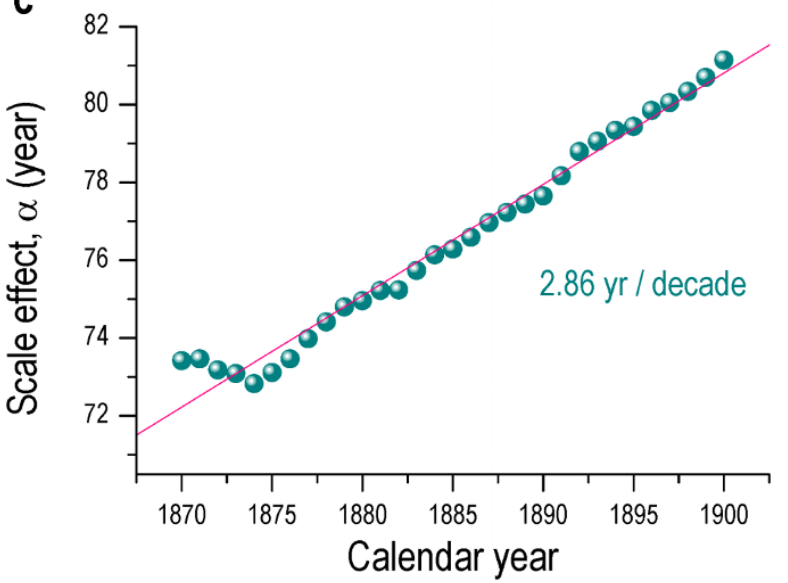

b

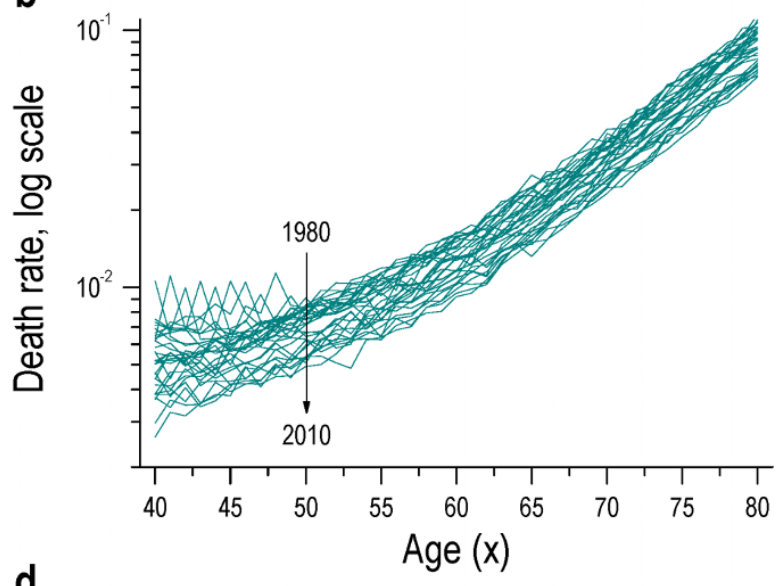

d

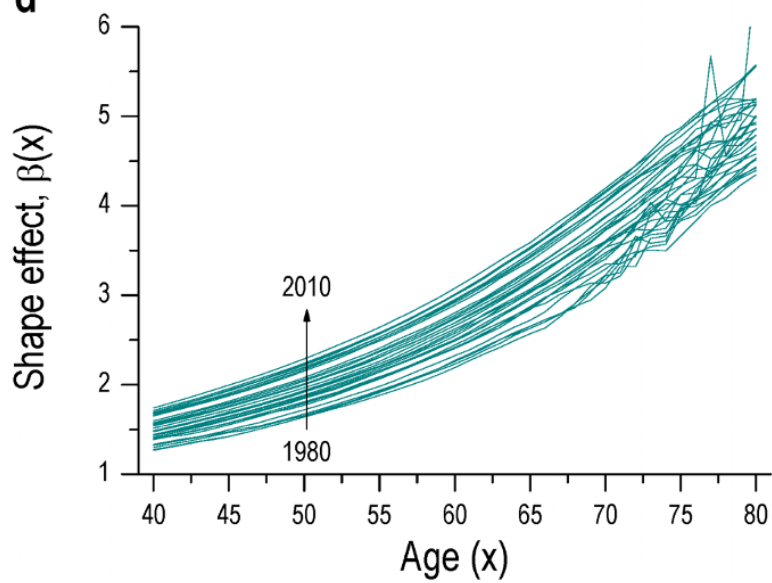

Figure 3 Historical trends for Swedish female cohort data. For comparison with period data (1980-2010; Fig. 2), we analysed cohort data (1870-1900; Fig. 3) for Swedish females. The lag between the two series is 110 years. (a) The trends in survival curves based on cohort data are substantially different from those based on period data (Fig. 2a). This difference is attributed to the difference in the populations represented by the period and cohort datasets. (b) The death rates at $40-80$ years for cohort data show a nonlinear increase in $\ln (\mu(x))$ with age. The patterns shown by these death rates would be more adequately fitted by the Gompertz-Makeham model, which modifies the Gompertz law by adding an age-independent mortality component to the age-dependent (Gompertz) component. (c) The scale effect $(\alpha)$ increases over time at a constant rate of 2.86 years per decade from 1870 to 1900 for the Swedish female cohort data. (d) The shape effect, $\beta(x)$, shows a nonlinear increase with age, similar to the nonlinear increase in $\ln (\mu(x))$ with age (b). Despite the differences between the population datasets, the death rates are clearly coupled with the survival rates and are susceptible to the shape effect in the survival curves.

each death rate curve from 1980 to 2010 (Supplementary Table S2). The regression results, supported by high linearity (most $R^{2}>0.99 ; R^{2}$ $=$ the coefficient of determination), show that the intercept linearly decreases with the calendar year, whereas the slope is nearly invariant during the most recent three decades (Supplementary Fig. S1). This result implies that the Gompertz law holds. Note that the death rate curve shows a continuous downwards shift in parallel with the calendar year.

The scale effect $(\alpha)$ in survival rates increases over time at a constant rate of 1.30 years per decade from 1980 to 2010 for the Swedish female period data (Fig. 2c). The shape effect, $\beta(x)$, at $40-80$ years increases linearly with age (Fig. $2 \mathrm{~d}$ ). This trend strongly resembles the linear growth in $\ln (\mu(x))$ with age (Fig. 2b) except for the shift direction: "upwards" in $\beta(x)$ and "downwards" in $\ln (\mu(x))$. The age dependency of $\beta(x)$ at $40-80$ years can be fitted by the linear form $\beta(x)=\beta_{0}+\beta_{1} x$ at $x / \alpha<1$ (ref. 14). For comparison with the Gompertz law, we measured the intercept $\left(\beta_{0}\right)$ and slope $\left(\beta_{1}\right)$ for each $\beta(x)$ curve from 1980 to 2010 with high linearity (mostly $R^{2}>0.98$ ) (Supplementary Table S2).

The parallel shifts in the death rates during recent decades are associated with a violation of the Strehler-Mildvan law ${ }^{10}$. This law describes a negative linearity in the Gompertz parameters as $\ln (a)=\mathrm{C}+\mathrm{D} b$, where $\mathrm{C}=\ln (\mathrm{K})$ and $\mathrm{D}=-\mathrm{B}^{-1}$ are constants ${ }^{9}$. We indeed find a negative correlation between $\ln (a)$ and $b$ with $\mathrm{C}=-0.50501 \pm 1.03856, \mathrm{D}=$
$-106.93886 \pm 10.58095$ and $R^{2}=0.7789$ (based on data in Supplementary Table S2). This result leads to $\mathrm{K}=0.6035$ and $\mathrm{B}=$ 0.0094 and agrees well with other estimates of $K(0.05 \sim 0.15)$ and $B$ $(0.0092 \sim 0.0137)$ for Swedish female period data $^{10}$. From the high linearity in $\beta(x)$ with age $(x)$, we find a negative correlation between $\beta_{0}$ and $\beta_{1}$ as $\beta_{0}=\mathrm{E}+\mathrm{F} \beta_{1}$ with $\mathrm{E}=10.85398 \pm 0.85055, \mathrm{~F}=$ $-92.57897 \pm 7.91721$, and $R^{2}=0.8250$ (based on data in Supplementary Table S2). The historical trends in $\ln (\mu(x))$ with age suggest that the parallel shifts in $\ln (\mu(x))$ with calendar year would originate from the near-invariance in the coefficient $(b)$, whereas the negative linear correlation between $\ln (a)$ and $b$ would result from the continuous decrease in the intercept $(\ln (a))$ with the calendar year (Supplementary Fig. S2). The strong correlation between $\beta(x)$ and $\ln (\mu(x))$ implies that the death rates are coupled with the survival rates and are particularly susceptible to the shape effect in the survival curves.

Implications from cohort data in Sweden. We compare period data (1980-2010; Fig. 2) with cohort data (1870-1900; Fig. 3) for Swedish females. The lag between the two series is 110 years, corresponding to the maximum age in the life table. The difference between the period and cohort data follows from the computation of life tables: period life tables indicate the current death probability for people of different ages in a current year, whereas cohort life tables indicate the death probability of people from a given birth cohort. Note that 
a
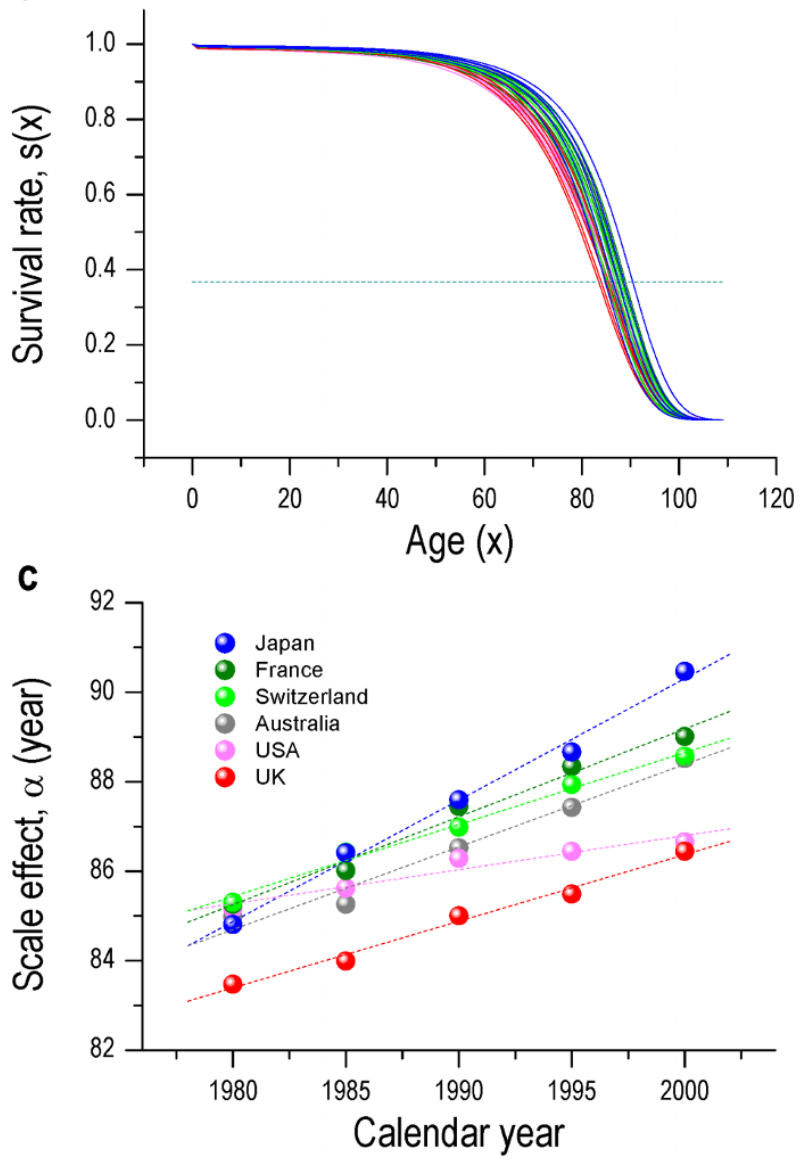

b

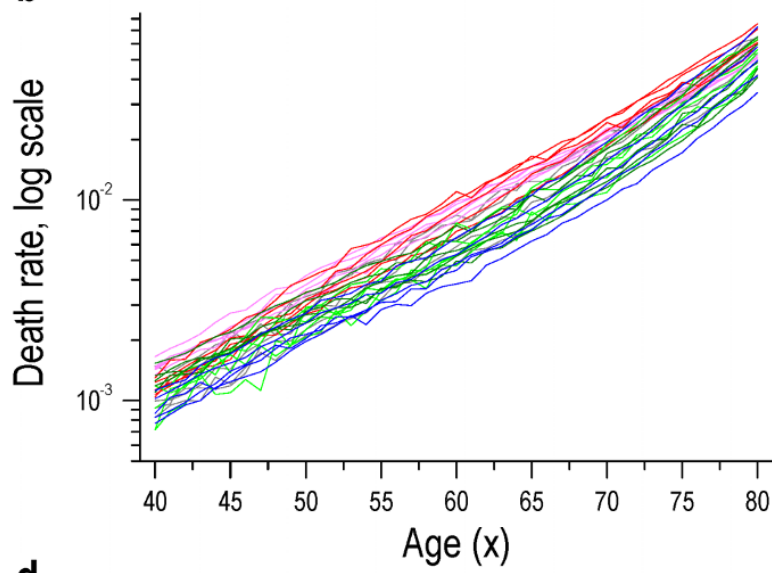

d

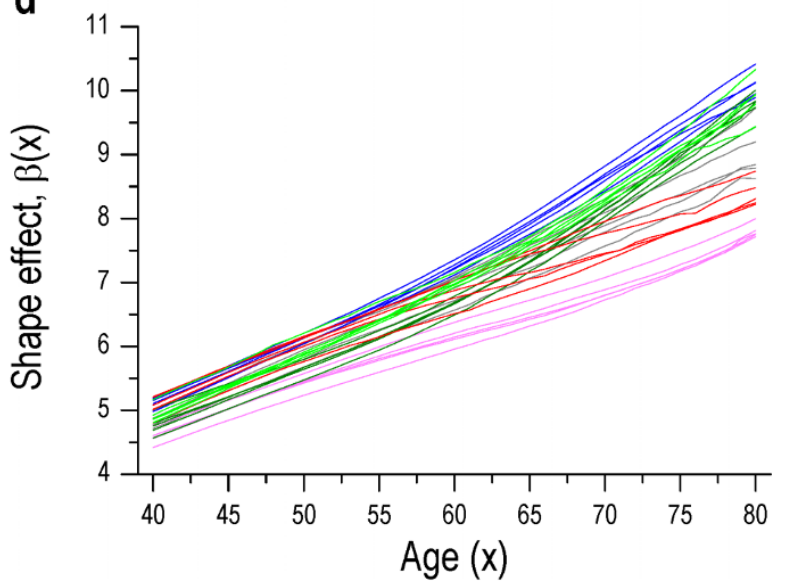

Figure $4 \mid$ Historical trends for selected wealthy countries. Demographic data from female period life tables from 1980 to 2000 (interval = 5 years) for Japan, France, Switzerland, Australia, the USA, and the UK were selected for wealthy countries to demonstrate the differences among these countries. (a), (b) The differences in survival rates (a) and death rates at 40-80 years (b) by country are indicated by different colours. The patterns of survival and death rates do not appear to vary substantially by country. (c), (d) Scale and shape analyses of survival curves, measured by $\alpha$ (c) and $\beta(x)$ (d), respectively. We find that these patterns differ substantially among countries. In particular, the characteristic life increases over time at very different rates of increase in different countries. Japan shows the highest rate and the USA the lowest rate. France, Switzerland, and Australia show trends similar to Japan but differ from the USA and the UK. Divergent trends in the shape effect by country influence the divergence in the scale effect for older ages ( $>60$ years).

the trends in the survival curves from the cohort data (Fig. 3a) differ markedly from the period data (Fig. 2a). This outcome can be attributed to the difference between the populations represented by the period and cohort datasets. The death rates at $40-80$ years (Fig. 3b) for the cohort data do not show a simple exponential increase with age. The patterns shown by these death rates would be more adequately fitted by the Gompertz-Makeham model, which modifies the Gompertz law by adding an age-independent mortality component to the age-dependent (Gompertz) component (see, for instance, ref. 23). The scale effect $(\alpha)$ increases over time at a constant rate of 2.86 years per decade from 1870 to 1900 for the Swedish female cohort data (Fig. 3c; Supplementary Table S3). Note that the shape effect, $\beta(x)$, shows a nonlinear increase with age (Fig. 3d), similar to the nonlinear increase in $\ln (\mu(x)$ ) (Fig. 3b). Despite the differences between the population datasets, it is clear that the death rates are coupled with the survival rates and are susceptible to the shape effect in the survival curves.

Implications from worldwide data for wealthy countries. Additionally, we analyse the scale and shape trends of survival curves for selected wealthy countries (Japan, France, Switzerland, Australia, the USA, and the UK) for female period life tables from 1980 to 2000 (interval $=5$ years). The differences in the survival and death rates by country are illustrated by different colours in Figs. $4 \mathrm{a}$ and $4 \mathrm{~b}$, respectively. The patterns of the survival and death rates do not appear to vary substantially by country. However, the scale and shape analyses of the survival curves, measured by $\alpha$ and $\beta(x)$ in Figs. $4 \mathrm{c}$ and $4 \mathrm{~d}$, respectively, show that these patterns differ markedly among countries. In particular, the characteristic life increases over time at very different rates of increase in different countries (Supplementary Table S3). Japan shows the highest rate and the USA the lowest rate. France, Switzerland, and Australia show trends similar to Japan but differ from the USA and the UK. We find that the divergent trends in the shape effect by country are responsible for the divergence in the scale effect for older ages ( $>60$ years). Our analyses, based on scale and shape variations in the survival curves, can provide useful insights into ageing trends.

\section{Discussion}

Current demographic trends deserve careful attention because they furnish clues that help explain the dynamic features of human ageing. In recent decades, modern civilisation has achieved substantial reductions in human mortality ${ }^{24-26}$. The global female life expectancy has shown a steady increase, with a slope of 0.243 , from 1840 to $2001^{25,26}$. Most demographers expect that mortality trends will continue to decline and that life expectancy will continue to increase, with no limit yet evident, as forecasted for countries such as Japan and Sweden ${ }^{27}$. The precise tracking of mortality rates is important for global health policy because mortality changes substantially across 
countries and over time ${ }^{28,29}$. The recent mortality trends should be monitored and analysed carefully because of the possibility of identifying ageing mechanisms (see, for instance, ref. 30). This study suggests that human mortality trends may be analysed effectively by tracking the scale and shape variations of survival curves.

In this study, we describe a useful methodology that enables us to separately measure the scale and shape variations of survival curves. This information is important in determining whether humans are living longer (scale trends) and growing older (shape trends). Indeed, human survival curves vary in terms of scale and shape over time in different population datasets as a result of various factors. Our analyses suggest that death rates are coupled with survival rates and are much more susceptible to the shape effect of survival curves than to the scale effect. If the scale effect constantly increases over time, the correlation between death rate trends and shape effect trends in survival curves would be strong because death rates are susceptible to the shape effect in survival curves. Furthermore, identifying the causes of mortality trends is an important issue in ageing statistics and in studies on the biological origins of ageing ${ }^{31-33}$. We believe that scale and shape analyses of survival curves will be useful for identifying the causes of the current trends in human ageing by providing quantification and connectivity between trends in scale and shape from human survival curves and by determining the possible factors influencing these trends. Complex relationships between life and death processes could be relevant to many biological and nonbiological factors involved in human survival and in the dynamics of ageing (see additional discussion in Supplementary Information). Further study is needed to identify the biological bases that are relevant to our analysis and the relevance to other demographic models.

Note that alternative explanations can be suggested based on the Gompertz-Makeham model, $\mu(x)=c+a \exp (b x)$, where $c$ is an ageindependent component ${ }^{30}$. In terms of the shape factor, the rectangularisation of the survival curve can be explained (i) by decreasing the rate of extrinsic or accidental mortality factors (captured by parameter $c$ if $a$ and/or $b$ are constant) and (ii) by the Strehler-Mildvan correlation if the initial vulnerability of the population (captured by parameter $a$ ) decreases in association with an increase in $b^{30}$. The former explanation is relevant to the period before 1950; the second is relevant to the period after 1950, when the $c$ in Sweden became too small for further variation to contribute significantly to changes in mortality patterns $^{30}$. The Strehler-Mildvan correlation is still evident in intercountry comparisons under conditions of negligibly small $c$ values ${ }^{34}$. If $c$ and its changes are significant, the use of the Gompertz model $(c=$ 0 ) may lead to an artefactual Strehler-Mildvan correlation ${ }^{30}$. In terms of the scale factor, an increasing population or cohort lifespan has the following alternative explanations: (i) an increase in population size being exhausted by mortality, as shown for Sweden ${ }^{35}$; (ii) the associated increase in the probability that an individual will survive to older ages by chance; and (iii) a more protected environment and medical advances that extend the lives of the oldest ${ }^{36}$.

Ageing is an inevitable fact of life ${ }^{37}$. The survival rate is typically used by molecular biologists to measure the life span or the ageing rate, while the mortality rate is used by demographers, epidemiologists, and evolutionary biologists ${ }^{37}$. It is believed that ageing occurs because natural selection is weak and ineffective at maintaining survival, reproduction, and somatic repair at old age ${ }^{38}$. This study demonstrates that scale and shape variations in survival curves can be measured simply with our methodology despite the complexity in the details of the dynamics of life and death. Our methodology, based on scale and shape analyses of survival curves, can contribute significantly to the improved tracking and forecasting of ageing statistics.

\section{Methods}

Survival and death rate data from female period $(1 \times 1)$ or cohort life tables $(1 \times 1)$ for selected wealthy countries (Sweden, Japan, France, Switzerland, Australia, the USA, and the UK) were separately taken from the Human Mortality Database (http:// www.mortality.org). The characteristic life $(\alpha)$ values, indicating the scale effect of survival curves, were measured from each survival curve through graphical analysis at $s(\alpha)=\exp (-1) \approx 0.367879$ (a standard deviation in measurements $\approx 3 \times 10^{-5}$ ). The age-dependent stretched exponent, which indicates the shape effect of survival curves, was obtained by computing $\beta(x)=\ln [-\ln (s(x))] / \ln (x / \alpha)$. A comparison between the characteristic life and the life expectancy at birth for Swedish women (from the Human Mortality Database) was made (Supplementary Fig. S1). Simple linear regressions for the middle ages of 40-80 years were performed with $\ln (\mu(x))=\ln (a)$ $+b x$ and $\beta(x)=\beta_{0}+\beta_{1} x$ for Swedish female period data (Supplementary Table 2). The parameter correlations and the historical trends in $b, \ln (a), \beta_{1}$, and $\beta_{0}$ were analysed with $\ln (a)=\mathrm{C}+\mathrm{D} b$ and $\beta_{0}=\mathrm{E}+\mathrm{F} \beta_{1}$ for the period data of 1980-2010 (based on the data in Supplementary Table 2 and Supplementary Fig. S2).

1. Vaupel, J. W. Biodemography of human ageing. Nature 464, 536-542 (2010).

2. Kirkwood, T. B. L. \& Melov, S. On the programmed/non-programmed nature of ageing within the life history. Curr. Biol. 21, R701-R707 (2011).

3. Partridge, L. \& Mangel, M. Messages from mortality: the evolution of death rates in the old. Trends Ecol. Evol. 14, 438-442 (1999).

4. Metcalf, C. J. E. \& Pavard, S. Why evolutionary biologists should be demographers. Trends Ecol. Ecol. 22, 205-212 (2006).

5. Hawkes, K., Smith, K. R. \& Blevins, J. K. Human actuarial aging increases faster when background death rates are lower: a consequence of differential heterogeneity? Evolution 66, 103-114 (2012).

6. Gompertz, B. On the nature of the function expressive of the law of human mortality. Phil. Trans. R. Soc. Lond. A 115, 513-580 (1825).

7. Guarente, L. Link between aging and the nucleolus. Genes Dev. 11, 2449-2455 (1997).

8. Mueller, L. D. \& Rose, M. R. Evolutionary theory predicts late-life mortality plateaus. Proc. Natl. Acad. Sci. USA 93, 15249-15253 (1996).

9. Strehler, B. L. \& Mildvan, A. S. General theory of mortality and aging. Science 132, 14-21 (1960).

10. Yashin, A. I., Begun, A. S., Boiko, S. I., Ukraintseva, S. V. \& Oeppen, J. New age patterns of survival improvement in Sweden: do they characterize changes in individual aging? Mech. Ageing Dev. 123, 637-647 (2002).

11. Yashin, A. I., Begun, A. S., Boiko, S. I., Ukraintseva, S. V. \& Oeppen, J. The new trends in survival improvement require a revision of traditional gerontological concepts. Exp. Gerontol. 37, 157-167 (2001).

12. Weon, B. M. \& Je, J. H. Theoretical estimation of maximum human lifespan. Biogerontology 10, 65-71 (2009).

13. Weon, B. M. \& Je, J. H. Predicting human lifespan limits. Natural Science 2, 984989 (2010).

14. Weon, B. M. \& Je, J. H. Plasticity and rectangularity in survival curves. Scientific Reports 1, 104 (2011).

15. Kohlrausch, R. Theorie des elektrischen rückstandes in der leidener flasche. Pogg Ann. Phys. Chem. 91, 179-214 (1854); Williams, G. \& Watts, D. C. Nonsymmetrical dielectric relaxation behavior arising from a simple empirical decay function. Trans. Faraday Soc. 66, 80-85 (1970).

16. Weibull, W. A. A statistical distribution function of wide applicability. J. Appl. Mech. 18, 293-297 (1951).

17. Piantanelli, L. et al. Use of mathematical models of survivorship in the study of biomarkers of aging: the role of heterogeneity. Mech. Ageing Dev. 122, 1461-1475 (2001).

18. McMichael, A. J., McKee, M., Shkolnikov, V. \& Valkonen, T. Mortality trends and setbacks: global convergence or divergence? Lancet 363, 1155-1159 (2004).

19. Lee, R. Sociality, selection, and survival: simulated evolution of mortality with intergenerational transfers and food sharing. Proc. Natl. Acad. Sci. USA 105, 7124-7128 (2008).

20. Milne, E. M. The natural distribution of survival. J. Theor. Biol. 255, 223-236 (2008).

21. Olshansky, S. J. The law of mortality revisited: interspecies comparisons of mortality. J. Comp. Pathol. 141, S4-S9 (2010).

22. Lee, R. D. Rethinking the evolutionary theory of aging: transfers, not births, shape senescence in social species. Proc. Natl. Acad. Sci. USA 100, 9637-9642 (2003).

23. Golubev, A. Does Makeham make sense? Biogeronology 5, 159-167 (2004).

24. Wilmoth, J. R. The future of human longevity: a demographer's perspective. Science 280, 395-397 (1998).

25. Oeppen, J. \& Vaupel, J. W. Demography. Broken limits to life expectancy. Science 296, 1029-1031 (2002).

26. Christensen, K., Doblhammer, G., Rau, R. \& Vaupel, J. W. Ageing populations: the challenges ahead. Lancet 374, 1196-208 (2009).

27. Lee, R. The outlook for population growth. Science 333, 569-573 (2011).

28. Mathers, C. \& Boerma, T. Mortality measurement matters: improving data collection and estimation methods for child and adult mortality. PLoS Med. 7, e1000265 (2010).

29. Rajaratnam, J. K., Marcus, J. R., Levin-Rector, A., Chalupka, A. N., Wang, H., Dwyer, L., Costa, M., Lopez, A. D. \& Murra, C. J. L. Worldwide mortality in men and women aged 15-59 years from 1970 to 2010: a systematic analysis. Lancet 375, 1704-1720 (2010).

30. Gavrilov, L. A. \& Gavrilova, N. S. The Biology of Life Span: A Quantitative Approach (Harwood Academic Publisher: New York, 1991).

31. Wang, S., Szalay, M. S., Zhang, C. \& Csermely, P. Learning and innovative elements of strategy adoption rules expand cooperative network topologies. PLoS ONE 3, e1917 (2008).

32. Kiss, H. J. M. et al. Ageing as a price of cooperation and complexity. BioEssays 31, 651-664 (2009). 
33. Schrodinger, E. What is Life (Cambridge University Press: Cambridge, 1944).

34. Golubev, A. How could the Gompertz-Makeham law evolve. J. Theor. Biol. 258, 1-17 (2009).

35. Wilmoth, J. R., Deegan, L. J., Lundstrom, H. \& Horiuchi, S. Increase of maximum life-span in Sweden, 1861-1999. Science 289, 2366-2368 (2000).

36. Finkelstein, M. Discussing the Strehler-Mildvan model of mortality. Demographic Research 26, 191-206 (2012).

37. Bronikowski, A. M. \& Flatt, T. Aging and its demographic measurement. Nature Education Knowledge 1, 3 (2010).

38. Fabian, D. \& Flatt, T. The evolution of aging. Nature Education Knowledge 3, 9 (2011).

\section{Acknowledgements}

This research was supported by the Creative Research Initiatives (Functional X-ray

Imaging) of the Ministry of Education, Science, and Technology of Korea (MEST) and the National Research Foundation of Korea (NRF).

\section{Author contributions}

B.M.W. conceived, designed, and performed the analyses and was the primary author of the manuscript. J.H.J. contributed to the discussion and the final manuscript.

\section{Additional information}

Supplementary information accompanies this paper at http://www.nature.com/ scientificreports

Competing financial interests: The authors declare no competing financial interests.

License: This work is licensed under a Creative Commons

Attribution-NonCommercial-ShareAlike 3.0 Unported License. To view a copy of this license, visit http://creativecommons.org/licenses/by-nc-sa/3.0/

How to cite this article: Weon, B.M. \& Je, J.H. Trends in scale and shape of survival curves. Sci. Rep. 2, 504; DOI:10.1038/srep00504 (2012). 